\title{
Novel Sample Preparation for Operando TEM of Catalysts
}

\author{
Benjamin K Miller ${ }^{[1]}$, Trevor M Barker ${ }^{[1]}$, Peter A Crozier ${ }^{[1]^{*}}$ \\ ${ }^{[1]}$ School for Engineering of Matter, Transport and Energy, \\ Arizona State University, Tempe, AZ 85287-6106 USA
}

*Corresponding author: Peter A Crozier

Engineering G Wing, \#301

$501 \mathrm{E}$. Tyler Mall

Tempe, AZ 85287-6106

Tel: 4809652934

Fax: 4807279321

Email: crozier@asu.edu

Keywords

Operando

In Situ TEM

Environmental TEM (ETEM)

TEM Sample

EELS

Catalyst 


\section{Abstract}

A new TEM sample preparation method is developed to facilitate operando TEM of gas phase catalysis. A porous Pyrex-fiber pellet TEM sample was produced, allowing a comparatively large amount of catalyst to be loaded into a standard Gatan furnace-type tantalum heating holder. The increased amount of catalyst present inside the environmental TEM allows quantitative determination of the gas phase products of a catalytic reaction performed in-situ at elevated temperatures. The product gas concentration was monitored using both electron energy loss spectroscopy (EELS) and residual gas analysis (RGA). Imaging of catalyst particles dispersed over the pellet at atomic resolution is challenging, due to charging of the insulating glass fibers. To overcome this limitation, a metal grid is placed into the holder in addition to the pellet, allowing catalyst particles dispersed over the grid to be imaged, while particles in the pellet, which are assumed to experience identical conditions, contribute to the overall catalytic conversion inside the environmental TEM cell. The gas within the cell is determined to be wellmixed, making this assumption reasonable. 


\section{Introduction}

Linking catalyst microstructure to catalytic performance is a primary objective to advance the science of heterogeneous catalysis. To accomplish this, one must know the structure of a working catalyst when it is catalyzing a reaction of interest. Various methods have been applied to determine this structure using transmission electron microscopy (TEM). High surface area catalysts can be studied in the TEM, then used in a reactor and, after reaction, again observed in the microscope [1,2]. This post-mortem analysis can yield important information, but may not reveal the structures present during the reaction, as these may be transient, and exist only under reaction-conditions $[3,4]$. In-situ techniques attempt to solve this problem by imaging the sample while subjecting it to conditions that simulate a reactor $[5,6]$. This is a powerful technique, and many advances have been made recently allowing higher pressures over 1 atmosphere $[7,8]$, as well as rapid heating with minimal thermal drift of the sample $[9,10]$.

Despite these advances, the conditions within the microscope are still often different from conditions present in ex-situ reactors. Additionally, the temperature and gas composition inside the ETEM cell are sometimes not accurately known [11,12]. If the conditions are not precisely known or controlled, a catalyst which may be assumed to be active may actually be inactive and the link between catalyst structure and performance will be ambiguous. To address this problem it is necessary to measure the catalyst relative activity throughout the in-situ experiment. This is the goal of operando TEM. In an operando experiment, the gas composition within the microscope is measured $[13,14]$. This decreases the uncertainty regarding the gas composition around the sample. More importantly, it makes it possible to follow the course of the reaction, monitoring the changes in catalyst activity and structure simultaneously. Now the ensemble of structures observed in the TEM is unambiguously active for the reaction being studied whenever the expected gaseous products are observed. The correlation between the relative catalyst activity and the catalyst structure will now be much tighter. 
A traditional TEM sample has a very low surface area over which to disperse a catalyst powder, and the minute amount of catalyst inside the microscope will yield a correspondingly small number of product gas molecules. In a windowed cell setup, it may be possible to detect this small number of product gas molecules in the outlet flow from the holder, since the total flow is quite small [15]. In a differentially pumped environmental TEM (ETEM) [12] however, the reactant-gas flow is much larger, so the partial pressures of product gases will be smaller. If these partial pressures are near the detection limit of the equipment used to measure the gas composition, it will be difficult to accurately measure the catalyst's changing activity. A simple solution to this problem is to increase the amount of catalyst present in the TEM, so that an easily quantifiable amount of product gas is produced. This paper describes a new sample preparation technique to achieve this increase for a differentially pumped ETEM.

\section{Materials/Methods}

\subsection{Operando Sample Preparation}

The TEM sample developed for operando TEM is a combination of two distinct samples, simultaneously loaded into a single TEM holder, as depicted in Figure 1. The first part of the sample is a metal mesh grid over which a catalyst is dispersed. The second half of the sample is an inert porous operando sample pellet, made from Pyrex glass fibers, which is also covered with the same catalyst (CO conversion on the Pyrex and mesh was shown to be neglible). The TEM holder for which this sample was created is a Gatan furnace-style heating holder. This holder supports a standard $3 \mathrm{~mm}$ TEM sample inside a small furnace. A hex ring with exterior threads screws into the internally threaded furnace body, securing the sample in place. Both the grid and pellet must fit into this $0.75 \mathrm{~mm}$ deep furnace.

To produce the operando sample pellet, Pyrex fibers (about 80 microns in diameter) are first crushed in a small mortar and pestle so that the length of each fiber is on the order of a millimeter. This prevents self-alignment of fiber bundles, which was found to occur with long, uncrushed fibers. The crushed 
fibers are then packed into a $3 \mathrm{~cm}$ long quartz tube whose inner diameter is $3 \mathrm{~mm}$. The packed tube is then fired at $700^{\circ} \mathrm{C}$ for 3 hours, to initiate sintering of the fibers into a network. After cooling to room temperature, the resulting porous cylinder of networked fibers is pushed out of the quartz tube, and sliced into sections approximately 1-2 mm thick with a razor blade. One side of each section is ground flat with sandpaper before placing the section into a small jig designed for finishing the pellets. The jig, depicted in Figure 2c, consists of two steel parts with a $0.8 \mathrm{~mm}$ hole through the centers. One of the parts has a counter bore that is $0.6 \mathrm{~mm}$ deep to accommodate the pellet. The pellet is placed, flat surface down inside this counter bore, and the rough surface is ground to the level of the steel with sand paper, leaving a $0.6 \mathrm{~mm}$ thick pellet. The steel pieces are then put together, and a $0.8 \mathrm{~mm}$ drill bit bores a hole through the pellet, guided by the steel parts to the exact center. The finished pellet is rinsed on a filter paper with nano-pure water to flush out any residual loose fibers. Loading catalyst onto the porous pellet is accomplished by impregnation using a suspension of the catalyst in water (see Figure $2 \mathrm{~b}$ ). Single drops of this catalyst suspension are impregnated into the pellet and allowed to dry before the next drop is added. The pellets are kept at about $80^{\circ} \mathrm{C}$ during this process to decrease the drying time between drops. The loading can be varied as needed, but for the present work was on the order of $5 \mu \mathrm{g}$ of supported catalyst per pellet.

An operando pellet produced by the method just described is seen in Figure 2a. The pellets are approximately $3 \mathrm{~mm}$ in diameter and $0.6 \mathrm{~mm}$ thick, and weigh approximately $3 \mathrm{mg}$ each, though there is some variation from one pellet to the next. The hole in the pellet is large enough that it does not place any additional restrictions on the maximum tilt angle achievable using the holder which is $35^{\circ}$. A typical pyrex pellet has a porosity of about $70 \%$, and thus good gas permeability. A typical pyrex pellet also has a large surface area, which can be calculated by considering that the pellet is composed of cylindrical fibers of known density. The surface area of a cylinder (neglecting the end caps) is $2 \pi r L$, while the mass of the cylinder is $\pi r^{2} L \rho$. Combining these two equations yields the surface area in terms of the mass, 
fiber radius, and density. This approach gives a surface area of approximately $7 \mathrm{~cm}^{2}\left(0.2 \mathrm{~m}^{2} / \mathrm{g}\right)$ for the pellet because it is composed of long, thin fibers. This is about 50 times the surface area of a normal 200 mesh $3 \mathrm{~mm}$ TEM grid.

For operando experiments involving temperatures above $500^{\circ} \mathrm{C}$, Pyrex glass fibers should not be used, but should be replaced by quartz fibers. Fabrication of a quartz pellet is similar to that of the Pyrex pellets, except that the quartz tube packed with crushed fibers should be fired at $1100^{\circ} \mathrm{C}$ for 2 hours. Quartz pellets are also suitable for studying photocatalysts due to their superior UV light transmission.

\subsection{Operando Test Using Pellet Sample}

CO oxidation was chosen as an ideal reaction to demonstrate this new TEM sample preparation technique since the chemistry is simple, and the reaction well-studied. The particular catalyst employed was ruthenium supported on amorphous silica spheres, which is seen schematically in Figure 1. The spheres were produced in the lab by the Stober method [16] and are approximately $200 \mathrm{~nm}$ in diameter. The Ru particles supported on these $\mathrm{SiO}_{2}$ spheres are between 5 and $15 \mathrm{~nm}$ in diameter.

Operando microscopy was performed on a differentially-pumped FEI Tecnai F20 ETEM at Arizona State University, operated at 200kV. In the Tecnai, $\mathrm{CO}$ and $\mathrm{O}_{2}$ gases were mixed in a stoichiometric ratio in a 1 $\mathrm{L}$ tank at about 0.5 bar, and the pressure in the ETEM cell was regulated using a leak valve. An activated carbon filter was placed prior to the leak valve to prevent the Fe and Ni carbonyls found in the $\mathrm{CO}$ gas from entering the TEM. Both residual gas analysis (RGA) and electron energy-loss spectroscopy (EELS) were used to monitor the gas composition during ETEM experiments, as detailed elsewhere [14]. The temperature of the pellet and grid were controlled using a Gatan Ta heating holder. The experiment demonstrating charging effects when imaging catalyst directly on the porous pellet was performed on an aberration corrected Titan ETEM operated at $80 \mathrm{kV}$. 


\section{Results/Discussion}

Figure 3 shows core-loss EELS spectra of the carbon K-edge from the gas mixture inside the ETEM during $\mathrm{CO}$ oxidation. The $\pi^{*}$ peak from $\mathrm{C}$ in $\mathrm{CO}$ is clearly distinguishable from the corresponding peak from $\mathrm{CO}_{2}$.

[17]. Spectra were acquired with and without the operando pellet present, and with a stoichiometric ratio of $\mathrm{CO}$ and $\mathrm{O}_{2}$ at $300^{\circ} \mathrm{C}$. Quantification of these spectra (by the methods described in [14]), show that the conversion is enhanced significantly when using this sample preparation, with only $3 \%$ conversion observed without the pellet, but $44 \%$ conversion with the pellet. This significant increase in conversion makes quantification more precise, enabling the in-situ behavior of the catalyst to be followed with decreasing temperature down to the light-off temperature. Comparison of in-situ conversion data with ex-situ reactor studies has shown good agreement [18]. While absolute activity values were not determined, useful information was obtained, since we are able to explore how the conversion varies during a single operando experiment. Such relative kinetic data is relevant to catalysis, and similar operando TEM data have been published recently for Pt [15].

While it is clear that this operando pellet sample is necessary for using EELS to quantify gaseous products, an argument could be raised that by using mass spectrometry, significantly smaller quantities can be detected, eliminating the need for the pellet sample. Though residual gas analysis is capable of measuring small quantities, in practice it is found that, for a differentially pumped ETEM, background signals from the vacuum are large, making it difficult to quantify small changes accurately. This is demonstrated in Figure 4 where at a pressure of 2 Torr of $\mathrm{CO}$ and $\mathrm{O}_{2}$ and a temperature of $130^{\circ} \mathrm{C}$, no $\mathrm{CO}_{2}$ should be produced by the catalyst, yet a large signal is detected at $44 \mathrm{~m} / \mathrm{z}$. In addition to $\mathrm{CO}, \mathrm{O}_{2}$ and $\mathrm{CO}_{2}$, many other signals are seen in the background signal of the RGA with appreciable intensity. This background may come from a variety of sources. If the vacuum system was not baked just prior to an experiment, then some gas may be present from past experiments, or from small air leaks into the 
system. In addition, the tungsten RGA filament itself is known to generate $\mathrm{H}_{2} \mathrm{O} \mathrm{CO}$ and $\mathrm{CO}_{2} \mathrm{creating}$ peaks at 18,28 , and 44 respectively $[19,20]$. For each of these sources, fragments and isotopes of the primary contaminant gases produce additional much smaller peaks. While the background signals would make it difficult to accurately quantify small conversions within the TEM, the pellet sample yields a high conversion in the TEM, which is readily seen in the RGA data in the Figure 4 inset.

Silica-supported catalyst particles, which were dispersed on the operando pellet, were challenging to image in the TEM, since the pellet fibers are insulating and charge under the electron beam. Even at room temperature in vacuum, the disruption to the optics was so severe that no lattice fringes could be seen within particles that were in contact with the fiber pellet. A "good" image of a silica-spheresupported particle on a fiber at room temperature is shown in Figure $5 \mathrm{a}$. While the basic shape of the catalyst particle can be seen, no detail is discernable. The solution to this problem is to use a metal mesh, loaded into the holder with the pellet (Figure 1). Images are acquired from supported catalyst particles dispersed on a metal mesh, rather than those on the fiber pellet. An image obtained from such a particle in the Titan ETEM, at $480^{\circ} \mathrm{C}$ in 0.75 Torr $_{2}$, is shown in Figure $5 \mathrm{~b}$. Imaging on the metal mesh significantly improves the image quality, and lattice fringes are visible, allowing for identification of the phase of the particle, which in this image is Ru.

We have introduced the operando pellet to increase the conversion inside the microscope, and this is effective, yet the silica-sphere-supported particles dispersed over a metallic mesh are the ones actually imaged. This setup necessitates that the conditions be nearly identical for both the supported catalyst dispersed over the mesh and over the pellet. The temperature difference between the fiber pellet, and the metal mesh should be relatively small since they are both inside the furnace of the Gatan heating stage which controls the temperature. The gas composition should also be virtually identical, because the gases within the TEM sample chamber appear to be well mixed. 
Experimental evidence for a well-mixed composition was obtained by a simple experiment, summarized in Figure 6, using a pellet ground flat on 2 sides. Core-loss EELS was used to measure the gas composition at the center of the hole in a pellet during $\mathrm{CO}$ oxidation. The stage was then moved so that the gas composition was measured outside the pellet, beyond one of the ground edges. The gas composition measured with EELS was found to be identical in these two locations. At 1 Torr and $200^{\circ} \mathrm{C}$, the mean free path of the $\mathrm{CO}_{2}$ gas molecules is about $0.1 \mathrm{~mm}$, which is only one order of magnitude smaller than the distance between the sample and the pole-pieces. Thus, if the composition does not vary perpendicular to the beam direction, it is unlikely to vary significantly along the beam direction between the pole-pieces, and the gas around the sample will be well-mixed.

\section{Conclusion}

A new TEM sample preparation method is developed to facilitate operando TEM of gas phase catalysis in a differentially pumped ETEM. The catalytic conversion in the microscope has been significantly increased using the fiber pellet, lattice fringes of the catalyst particles can be resolved using a metallic grid, and the conditions at these two locations can be assumed to be nearly identical. These three achievements together should allow the structures observed in the TEM to be correlated with conversions measured in the microscope using both EELS and RGA. It is the goal of operando TEM studies to correlate some structural feature with a performance metric. This has been achieved through the development of a unique sample preparation technique for a supported catalyst sample.

\section{Acknowledgements}

We gratefully acknowledge helpful discussions with Patricia Kooyman as well as the use of facilities within the LeRoy Eyring Center for Solid State Science at Arizona State University. This work was supported by the US National Science Foundation (NSF-CBET 1134464). 

[1] J.M. Petroski, Z.L. Wang, T.C. Green, M.A. El-Sayed, Kinetically Controlled Growth and Shape Formation Mechanism of Platinum Nanoparticles, J. Phys. Chem. B. 102 (1998) 3316-3320. doi:10.1021/jp981030f.

[2] A.K. Datye, J. Bravo, T.R. Nelson, P. Atanasova, M. Lyubovsky, L. Pfefferle, Catalyst microstructure and methane oxidation reactivity during the $\mathrm{Pd} \leftrightarrow \mathrm{PdO}$ transformation on alumina supports, Appl. Catal. Gen. 198 (2000) 179-196. doi:10.1016/S0926-860X(99)00512-8.

[3] C.V. Ovesen, B.S. Clausen, J. Schiøtz, P. Stoltze, H. Topsøe, J.K. Nørskov, Kinetic implications of dynamical changes in catalyst morphology during methanol synthesis over $\mathrm{Cu} / \mathrm{ZnO}$ catalysts, J. Catal. 168 (1997) 133-142. doi:10.1006/jcat.1997.1629.

[4] P.L. Hansen, J.B. Wagner, S. Helveg, J.R. Rostrup-Nielsen, B.S. Clausen, H. Topsoe, Atom-Resolved Imaging of Dynamic Shape Changes in Supported Copper Nanocrystals, Science. 295 (2002) 20532055. doi:10.1126/science.1069325.

[5] R. Sharma, An Environmental Transmission Electron Microscope for in situ Synthesis and Characterization of Nanomaterials, J. Mater. Res. 20 (2005) 1695-1707. doi:10.1557/JMR.2005.0241.

[6] R. Sharma, Experimental set up for in situ transmission electron microscopy observations of chemical processes, Micron. 43 (2012) 1147-1155. doi:10.1016/j.micron.2012.01.007.

[7] J.F. Creemer, S. Helveg, G.H. Hoveling, S. Ullmann, A.M. Molenbroek, P.M. Sarro, et al., Atomicscale electron microscopy at ambient pressure, Ultramicroscopy. 108 (2008) 993-998. doi:10.1016/j.ultramic.2008.04.014.

[8] N. de Jonge, W.C. Bigelow, G.M. Veith, Atmospheric Pressure Scanning Transmission Electron Microscopy, Nano Lett. 10 (2010) 1028-1031. doi:10.1021/nl904254g.

[9] L.F. Allard, W.C. Bigelow, M. Jose-Yacaman, D.P. Nackashi, J. Damiano, S.E. Mick, A new MEMSbased system for ultra-high-resolution imaging at elevated temperatures, Microsc. Res. Tech. 72 (2009) 208-215. doi:10.1002/jemt.20673.

[10] J.F. Creemer, S. Helveg, P.J. Kooyman, A.M. Molenbroek, H.W. Zandbergen, P.M. Sarro, A MEMS Reactor for Atomic-Scale Microscopy of Nanomaterials Under Industrially Relevant Conditions, J. Microelectromechanical Syst. 19 (2010) 254-264. doi:10.1109/JMEMS.2010.2041190.

[11] S.B. Vendelbo, P.J. Kooyman, J.F. Creemer, B. Morana, L. Mele, P. Dona, et al., Method for local temperature measurement in a nanoreactor for in situ high-resolution electron microscopy, Ultramicroscopy. 133 (2013) 72-79. doi:10.1016/j.ultramic.2013.04.004.

[12] P.A. Crozier, S. Chenna, In situ analysis of gas composition by electron energy-loss spectroscopy for environmental transmission electron microscopy, Ultramicroscopy. 111 (2011) 177-185. doi:10.1016/j.ultramic.2010.11.005.

[13] S. Chenna, P.A. Crozier, Operando Transmission Electron Microscopy: A Technique for Detection of Catalysis Using Electron Energy-Loss Spectroscopy in the Transmission Electron Microscope, ACS Catal. 2 (2012) 2395-2402. doi:10.1021/cs3004853.

[14] B.K. Miller, P.A. Crozier, Analysis of Catalytic Gas Products Using Electron Energy-Loss Spectroscopy and Residual Gas Analysis for Operando Transmission Electron Microscopy, Microsc. Microanal. 20 (2014) 815-824. doi:10.1017/S1431927614000749.

[15] S.B. Vendelbo, C.F. Elkjær, H. Falsig, I. Puspitasari, P. Dona, L. Mele, et al., Visualization of oscillatory behaviour of Pt nanoparticles catalysing CO oxidation, Nat. Mater. 13 (2014) 884-890. doi:10.1038/nmat4033.

[16] W. Stöber, A. Fink, E. Bohn, Controlled growth of monodisperse silica spheres in the micron size range, J. Colloid Interface Sci. 26 (1968) 62-69. doi:10.1016/0021-9797(68)90272-5. 
[17] P.A. Crozier, S. Chenna, In situ analysis of gas composition by electron energy-loss spectroscopy for environmental transmission electron microscopy, Ultramicroscopy. 111 (2011) 177-185. doi:10.1016/j.ultramic.2010.11.005.

[18] S. Chenna, P.A. Crozier, Operando Transmission Electron Microscopy: A Technique for Detection of Catalysis Using Electron Energy-Loss Spectroscopy in the Transmission Electron Microscope, ACS Catal. 2 (2012) 2395-2402. doi:10.1021/cs3004853.

[19] J. Batey, Thermal desorption from mass spectrometer filaments, Vacuum. 43 (1992) 15-19. doi:10.1016/0042-207X(92)90176-W.

[20] J. O'Hanlon, A user's guide to vacuum technology, 3rd ed., Wiley-Interscience, Hoboken NJ, 2003. 


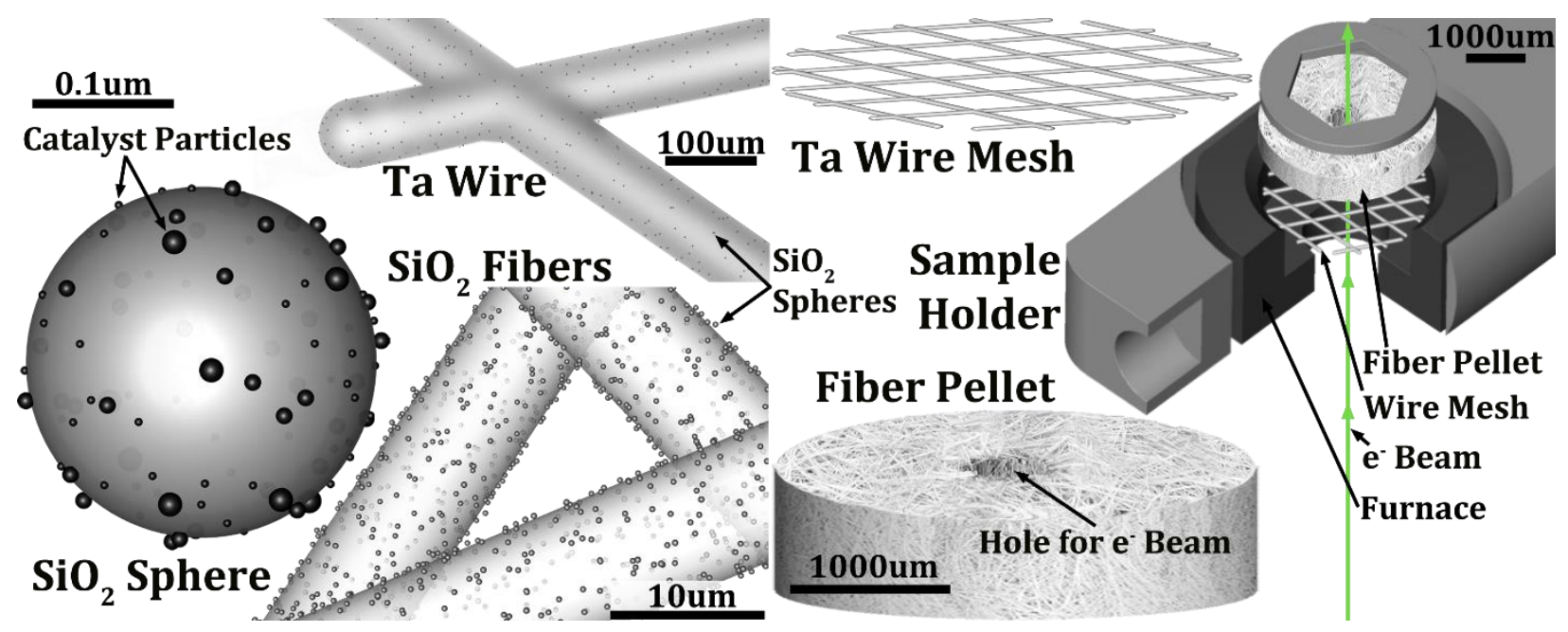

Figure 1 Operando TEM sample. An overview of the sample configuration used in the operando

experiments. Both a Ta wire mesh and a $\mathrm{SiO}_{2}$ fiber pellet are covered with silica-sphere-supported $\mathrm{Ru}$ catalyst particles, and placed inside the holder, with the wire mesh closer to the eucentric plane in the Ta heating holder. 


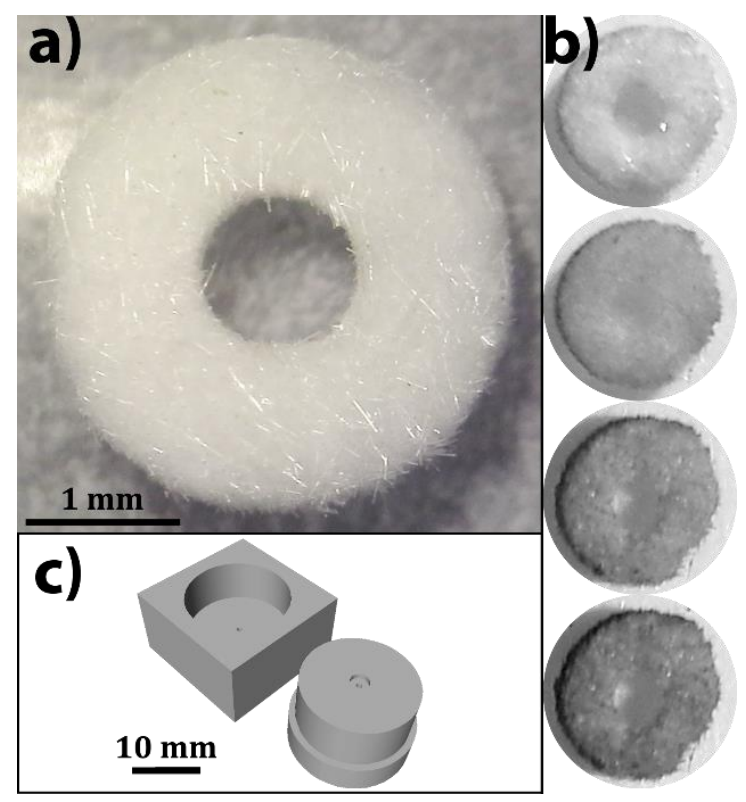

Figure 2 Operando Pellet. a) Optical images of the operando pellet, showing the texture and size. The hole in the center allows the electron beam to pass, and the wire mesh grid to be observed in the TEM. b) Series of images of an operando pellet after successive drops of a catalyst suspension have dried. c) The jig designed to finish the pellets, showing the small counter bore, in which the pellet sits, in the right half. 


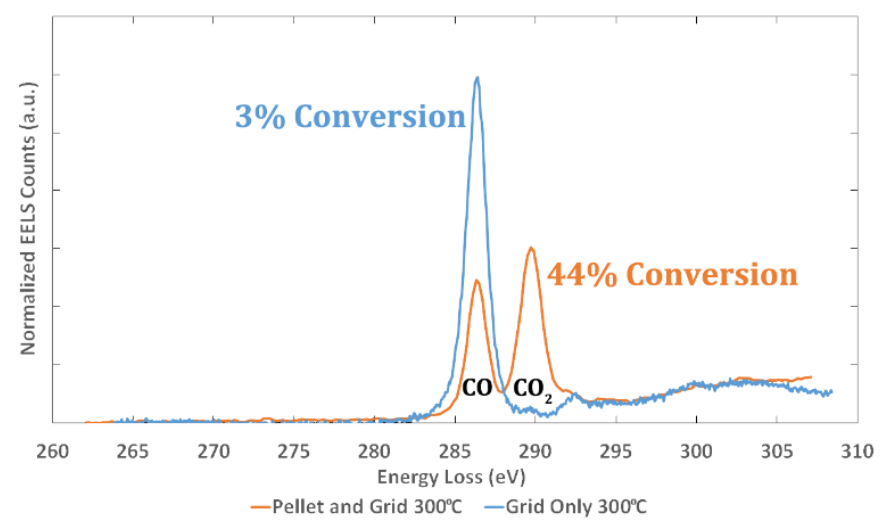

Figure $3 \mathrm{EELS}$ spectra taken at $300^{\circ} \mathrm{C}$ with a

stoichiometric mixture of $\mathrm{CO}$ and $\mathrm{O}_{2}$ in the cell. The

orange curve is from a pellet/grid sample, and the blue

from a standard TEM grid sample, showing significantly

increased conversion when using the pellet. 


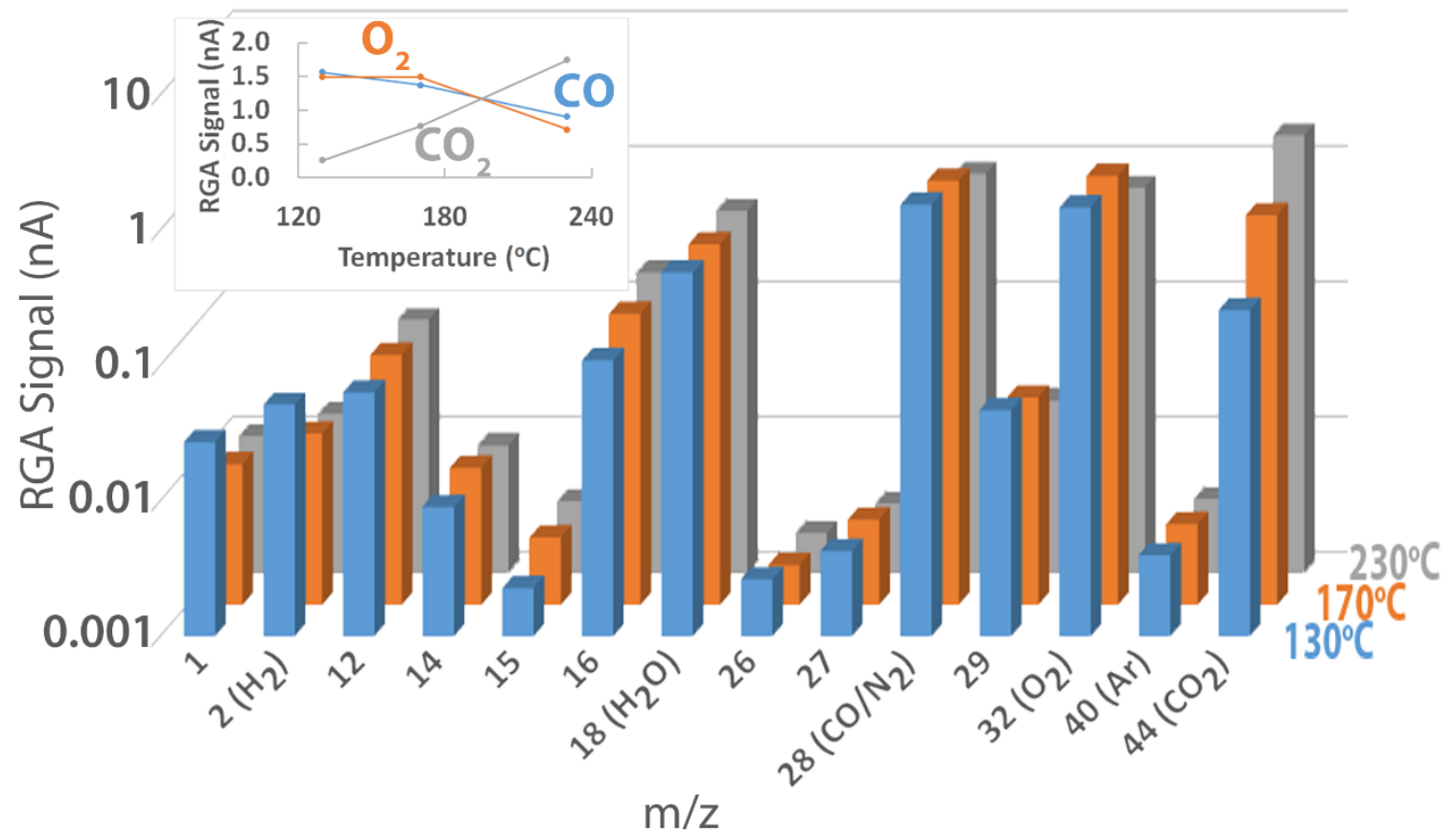

Figure 4 RGA Data. Residual gas analysis data showing the large background signal for $\mathrm{CO}_{2}$ and other gases at $130^{\circ} \mathrm{C}$ with a stoichiometric mixture of $\mathrm{CO}$ and $\mathrm{O}_{2}$ gas at 2 Torr in the ETEM cell. Inset: As the temperature is increased to $230^{\circ} \mathrm{C}$, the $\mathrm{CO}_{2}$ peak increases dramatically, due to the high conversion by the operando pellet. 


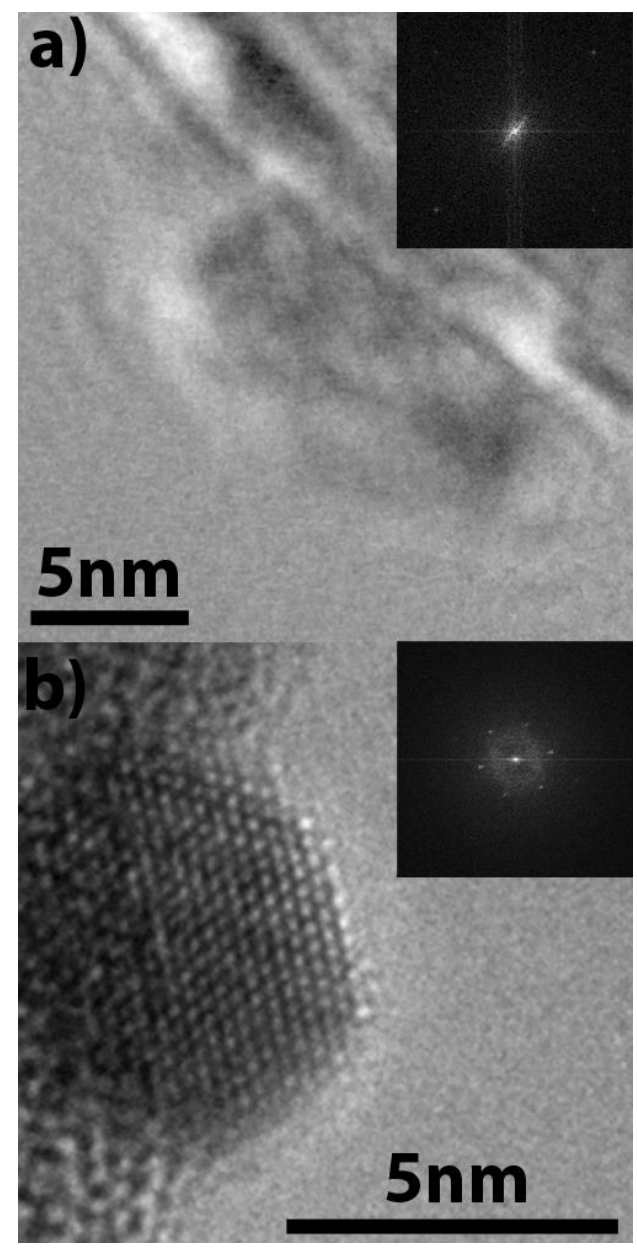

Figure 5 a) An image of a Ru particle supported on a silica sphere that is in contact with the insulating fiber pellet, showing the dramatic effect of charged fibers on the image resolution, even without gas and at room temperature. b) An image taken at $480^{\circ} \mathrm{C}$ in $0.75 \mathrm{Torr} \mathrm{H}_{2}$, from a particle supported on a silica sphere that is in contact with the Ta wire mesh, showing several lattice fringes clearly resolved. 


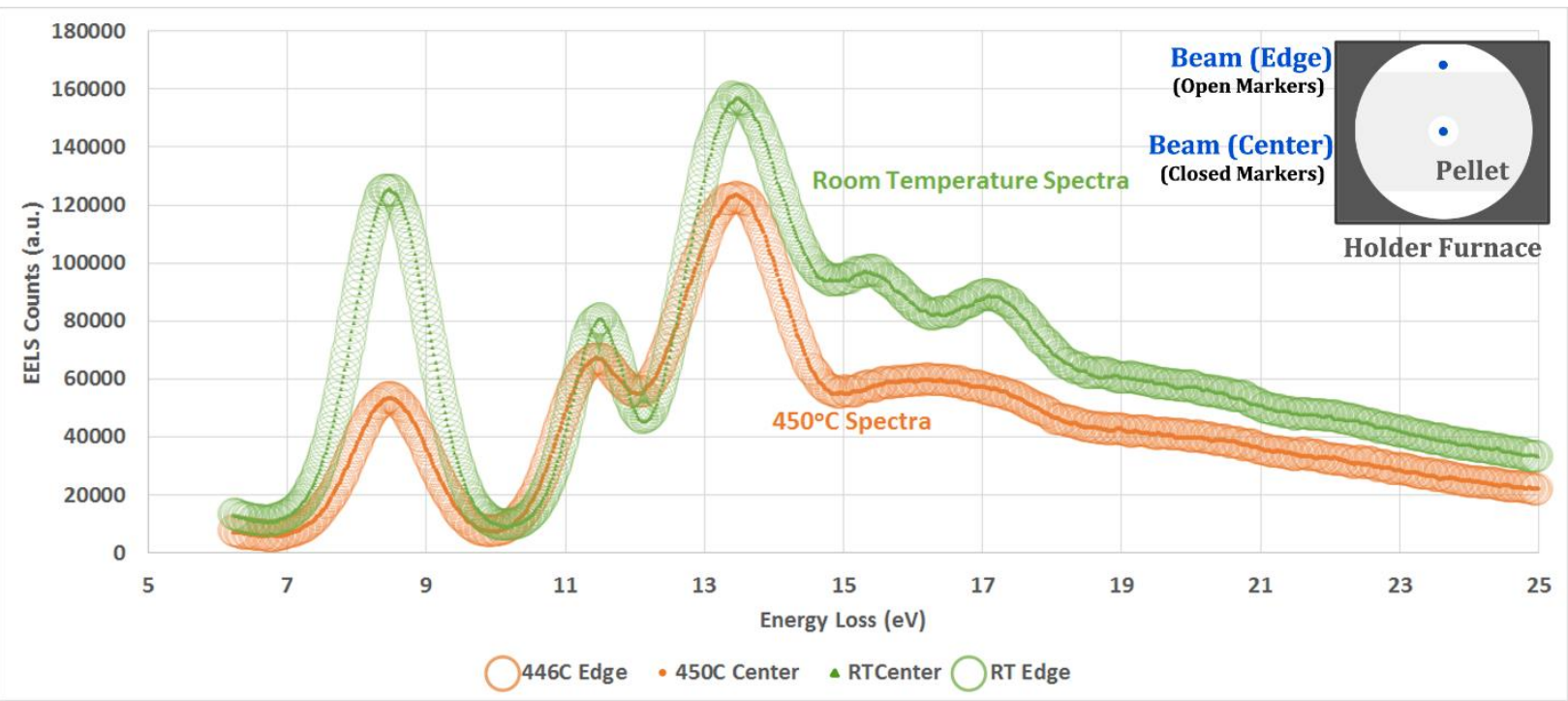

Figure 6 Gas Homogeneity. Four low-loss EELS spectra, showing that the gas composition at the outer edge of the fiber pellet is identical to the composition in the center of the pellet at both $450^{\circ} \mathrm{C}$ and room temperature, though the spectra at these two temperatures is significantly different due to high conversion at $450^{\circ} \mathrm{C}$. At each temperature, the large open symbols are the spectrum from the outside edge, while the small filled symbols are the spectrum from the center of the pellet. The inset shows the location of the measurements. 
